\title{
Estimating the Reduction of Radiated Emissions From Microstrip Components Using Network Analyzer With a Bulk Current Injection Probe
}

\author{
Cheng-Yu Ho, Student Member, IEEE, Kai-Syaun Chen, Student Member, IEEE, and \\ Tzyy-Sheng Horng, Senior Member, IEEE
}

\begin{abstract}
This work presents a novel measurement method using a network analyzer with a bulk current injection (BCI) probe to measure the common-mode conversion coefficient of microstrip components in an RF/microwave circuit. Based on the proposed measurement method, far-field radiated emissions from microstrip components are obtained, which closely corresponds to measurements in a fully anechoic chamber. The proposed method also estimates the radiated emission reduction by miniaturizing the physical size of microstrip bandpass filters (BPFs). Full-wave electromagnetic simulation further demonstrates the effectiveness of the measurement method.
\end{abstract}

Index Terms-Bulk current injection (BCI) probe, commonmode current, microstrip bandpass filter (BPF), network analyzer, radiated emissions.

\section{INTRODUCTION}

$\mathbf{R}$ ADIATED emissions from $\mathrm{RF} /$ microwave circuits are extremely complex and difficult to estimate. An RF/microwave circuit consists mainly of lumped elements, active devices, and microstrip components. Given that the dimensions of lumped elements and active devices are significantly smaller than those of microstrip components, radiated emissions from microstrip components are generally dominant than the others, thus posing a major challenge for electromagnetic compliance [1]. However, the radiated emissions from microstrip components on a printed circuit board (PCB) have seldom been studied largely owing to the predominance of common-mode radiation from cables attached to a $\mathrm{PCB}$. This circumstance has changed as the operating frequency has reached the gigahertz range, causing microstrip components on a PCB to radiate more efficiently. It is seen from an exhaustive literature review that a microstrip component may behave similar to an antenna in radiating the noise coupled from a microstrip amplifier on a PCB [1], [2]. Therefore, high-frequency PCB design must consider radiated emissions from the microstrip components.

Manuscript received October 01, 2012; accepted December 06, 2012. Date of publication January 18, 2013; date of current version February 08, 2013. This work was supported in part by the National Science Council of Taiwan under Grant 100-2622-E-110-001-CC1, Grant 100-2221-E-110-081-MY3, and Grant 100-2221-E-110-082-MY3.

The authors are with the Department of Electrical Engineering, National Sun Yat-Sen University, Kaohsiung 80424, Taiwan (e-mail: d943010018@student. nsysu.edu.tw; jason@ee.nsysu.edu.tw).

Color versions of one or more of the figures in this letter are available online at http://ieeexplore.ieee.org.

Digital Object Identifier 10.1109/LMWC.2012.2236887
Far-field radiated emissions can be measured in a fully-anechoic chamber (FAC), semi-anechoic chamber (SAC), or open area test site to obtain the spectra required in order to evaluate their compliance with electromagnetic interference (EMI) regulations [3]. However, such far-field measurements are prohibitively expensive and time consuming. Another conventional means of evaluating radiated emissions from a PCB involves a magnetic or electric field probe in which near-field scanning of the PCB surface is performed [4], [5]. Although the associated test setup and procedure are simpler than those of far-field measurements, the near-field scanning results are more effective in identifying the source of radiated emissions than in estimating radiated emission levels in line with EMI specifications. With the assistance of current probes, previous studies on PCB radiated emissions have characterized common-mode radiation from a specific power-ground plane structure with a cable attached [6], [7]. Far-field radiated emissions can thus be estimated based on the measured common-mode currents on a cable with a current probe. However, such measurements are limited to the power/ground planes with attached cables for estimating how they affect radiated emissions at low frequencies. In fact, PCBs for wireless communication products involve many microstrip components that radiate similar to an antenna at high frequencies, which still cannot be evaluated with current probe-based methods.

Network analyzer measurements have, in recent years, been incorporated in electromagnetic compatibility (EMC) applications to measure the $S$-parameters in correlation with the transfer function of a coupling path [8], [9]. Moreover, the bulk current injection (BCI) probe has been extensively adopted in standard electromagnetic susceptibility (EMS) testing [10]. Calibrating BCI probes with network analyzers has thus received considerable attention recently [11], [12]. Based on the above developments, this work presents a novel measurement method for evaluating common-mode radiation from microstrip components. Highly promising for use in EMI measurement application, the proposed method can estimate the reduction of radiated emissions by miniaturizing the physical size of the microstrip components on a PCB when pre-tested for compliance with EMI regulations.

\section{REduction OF COMMON-MODE CONVERSION COEFFICIENT}

Radiated emissions from PCB occur in very diverse ways. Experimental evidence suggests that EMI source and self-resonance of the circuit at the same frequency cause significant ra- 


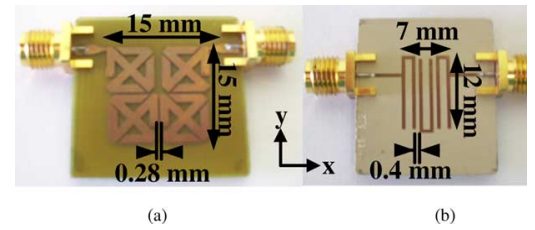

Fig. 1. Photograph and physical dimensions of microstrip BPFs. (a) Fourthorder net-type microstrip BPF. (b) Third-order hairpin-type microstrip BPF.

diated emissions at that frequency. In an RF/microwave circuit, microstrip component is a major radiation mechanism since its dimension is significantly larger than those of other discrete devices. Thus, the microstrip components on a PCB largely contribute to radiated emissions from the $\mathrm{RF} /$ microwave circuits.

Bandpass filters (BPFs) are crucial wireless components that suppress the output harmonics in a transmitter and input interferences in a receiver. Often constructed by coupling of resonators, their passband occurs close to resonant frequencies of the resonators, in which, electromagnetic radiation is easily emitted. Therefore, based on the proposed method, this work evaluates the radiated emissions from microstrip BPFs. Fig. 1(a) and (b) display the fourth-order net-type microstrip BPF [13] and the third-order hairpin-type microstrip BPF [14] that were implemented on a $0.8 \mathrm{~mm}$ thick FR4 substrate and a $0.64 \mathrm{~mm}$ thick Duroid substrate, respectively. Both microstrip $\mathrm{BPFs}$ were designed to have the same half-wavelength resonant frequency of approximately $2.4 \mathrm{GHz}$, in which the latter BPF has a smaller size than the former one.

Fig. 2 shows a microstrip BPF inserted into the center hole of the $\mathrm{BCI}$ probe to measure the common-mode conversion coefficients with a network analyzer. To verify measurement results of the common-mode conversion coefficient, the commonmode current based on the loop integral of the magnetic field around a microstrip BPF is investigated [15]. Fig. 3(a) and (b) show EM modeling of the closed-loop coil to determine the common-mode current of net- and hairpin-type microstrip BPF, respectively. The common-mode currents can thus be estimated in terms of the transmission coefficient $S_{21}$ and the BCI probe transfer impedance $Z_{T}$ as follows:

$$
I_{C M}(\omega)=\frac{V_{B C I}(\omega)}{Z_{T}(\omega)}=\frac{S_{21}(\omega)}{Z_{T}(\omega)} V_{i n}
$$

where $V_{\text {in }}$ denotes the input voltage wave amplitude of the microstrip BPF. The transmission coefficient $S_{21}$ in (1) can be treated as a conversion coefficient from microstrip mode to common mode. Notably, the BCI probe used in this work was manufactured by Fisher Custom Communications, Inc. with a model number F-150 [16]. Designed for operating frequencies ranging from 0.8 to $2.1 \mathrm{GHz}$, the $\mathrm{BCI}$ probe has been extended to operate up to $3 \mathrm{GHz}$ in this work. Therefore, the BCI probe is calibrated to determine the transfer impedance in the $0.8-3$ $\mathrm{GHz}$ frequency range. Consequently, the $\mathrm{BCI}$ probe has a frequency-dependent transfer impedance of 24-29 dB $\Omega$ across the entire calibration frequency range.

Fig. 4 compares the common-mode conversion coefficient obtained by the proposed method using a network analyzer with a BCI probe and by electromagnetic (EM) simulation using Ansys-Ansoft HFSS. Comparing the results from both

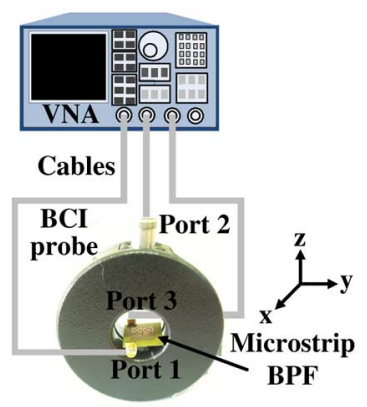

Fig. 2. Experimental setup for measuring common-mode conversion coefficient of the microstrip BPFs.

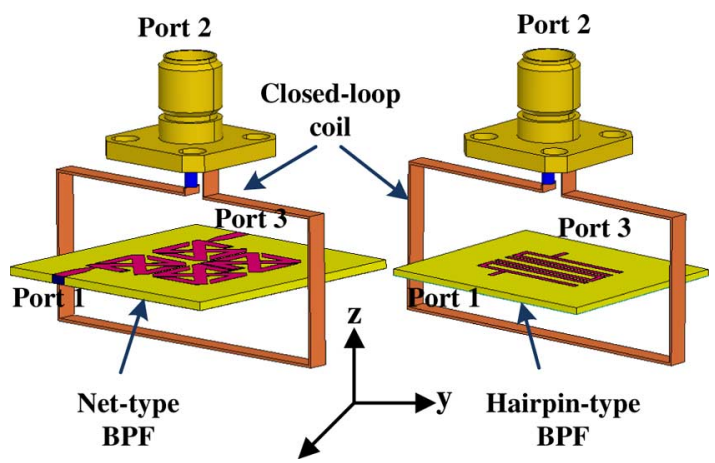

Fig. 3. EM simulation configurations for common-mode conversion coefficient of the microstrip BPFs.

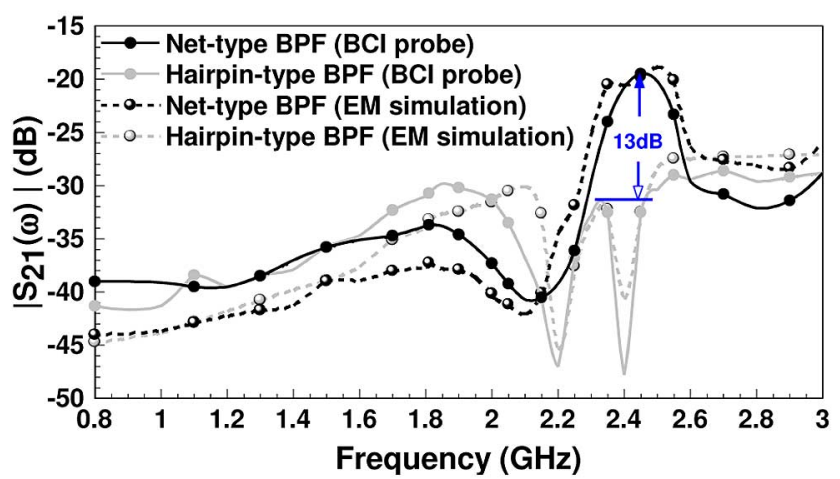

Fig. 4. Comparison of common-mode conversion coefficient magnitude of the net- and hairpin-type microstrip BPFs between the proposed method and the EM simulation.

approaches reveals a satisfactory correlation throughout the measurement frequency range. It is clearly seen from Fig. 4 that the net-type microstrip BPF has an approximately $13 \mathrm{~dB}$ higher maximum conversion coefficient than that of the hairpin-type microstrip BPF in the passband frequency range.

\section{Reduction of RAdiated EMISSIONS}

By assuming a uniform common-mode current distribution in a microstrip component, the far-field radiated emissions from the component can be estimated using the Hertzian dipole model, as given by [17]

$$
\left|E_{C_{\text {max }}}(\omega)\right|=4 \pi \times 10^{-7} \frac{I_{C M}(\omega) \cdot L \cdot \omega \cdot \cos \left(\frac{1}{2} \beta h\right)}{2 \pi R}
$$




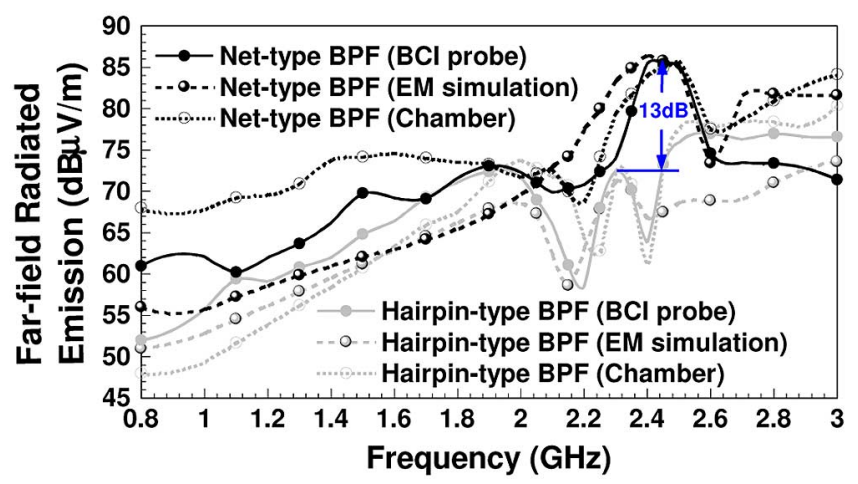

Fig. 5. Comparison of radiated emissions from the net- and hairpin-type microstrip BPFs obtained in a fully-anechoic chamber with predictions by the proposed method and EM simulation.

where $L$ denotes the length of the component, $h$ denotes the thickness of substrate, $\beta$ denotes the phase constant of free space, and $R$ denotes the distance between the measurement point and the component.

With (1) and (2), the $x$ - and $y$-directed common-mode current components on the microstrip BPF and their respective radiated emission in the far field are obtained. The overall radiated emissions are evaluated by taking the root of the sum of the square of each respective radiated emission. For validation, radiated emissions at $1 \mathrm{~m}$ distance are estimated by (2) at an input power of $0 \mathrm{dBm}$ (i.e., $V_{i n}=224 \mathrm{mV}$ ) and, then, compared with the FAC measurement results and the EM simulation results, as shown in Fig. 5. This comparison reveals a sufficient correlation in the passband frequencies of the microstrip BPFs. Notably, in this comparison, the input power to the microstrip BPFs is $0 \mathrm{dBm}$. According to this figure, the strongest radiation from the both microstrip BPFs occur in the passband owing to the use of netand hairpin-type resonators with a resonant frequency close to the passband. This figure also compares the results of radiated emissions between the net- and hairpin-type microstrip BPF. Coinciding with the difference of the common-mode conversion coefficients in Fig. 4, the former BPF has an approximately $13 \mathrm{~dB}$ higher maximum radiated emissions than the latter one in the passband. Miniaturizing the physical size of microstrip BPFs significantly reduces the far-field radiated emissions. Fig. 5 confirms the ability of the proposed common-mode conversion coefficient measurement method using a VNA with a $\mathrm{BCI}$ probe to accurately predict the far-field radiated emissions from the microstrip components on a PCB.

\section{CONCLUSION}

This work proposes a novel measurement method for using a network analyzer with a BCI probe to measure the common-mode conversion coefficient in order to characterize the common-mode radiation from a microstrip component. Additionally, the far-field radiated emission predictions obtained by the proposed method can confirm the contribution of a microstrip component to the EMI effect of a PCB when pre-tested for compliance with the EMI regulations. Furthermore, FAC measurements and EM simulations validate the accuracy of the proposed method. By using the proposed method, reduction of radiated emissions by miniaturizing the physical size of microstrip BPFs is evaluated. Results of radiated emission reduction obtained by the FAC measurements closely correspond to the predictions made by the common-mode conversion coefficients.

\section{REFERENCES}

[1] H. C. Hsieh, C. N. Chiu, C. H. Wang, and C. H. Chen, "A new approach for fast analysis of spurious emissions from $\mathrm{RF} /$ microwave circuits," IEEE Trans. Electromagn. Compat., vol. 51, no. 3, pp. 631-638, Aug. 2009.

[2] T. S. Horng and S. M. Wu, "Radiation from a microstrip amplifier," IEEE Trans. Microw. Theory Tech., vol. 50, no. 8, pp. 2005-2010, Aug. 2002.

[3] J. H. Kwon and H. D. Choi, "Experimental verification of correlation algorithm between FAC and open area test site/SAC," in IEEE Int. Symp. Electromagn. Compat. Dig., Aug. 2003, pp. 910-914.

[4] D. Baudry, C. Arcambal, A. Louis, B. Mazari, and P. Eudeline, "Applications of the near-field techniques in EMC investigations," IEEE Trans. Electromagn. Compat., vol. 49, no. 3, pp. 485-493, Aug. 2007.

[5] Y. Vives-Gilabert, C. Arcambal, A. Luis, F. de Daran, P. Eudeline, and B. Mazari, "Modeling magnetic radiations of electronic circuits using near-field scanning method," IEEE Trans. Electromagn. Compat., vol. 49, no. 2, pp. 391-400, May 2007.

[6] D. M. Hockanson, J. L. Drewniak, T. H. Hubing, and C. W. Lam, "Quantifying EMI resulting from finite-impedance reference planes," IEEE Trans. Electromagn. Compat., vol. 39, no. 4, pp. 286-297, Aug. 1997.

[7] D. M. Hockanson, J. L. Drewniak, T. H. Hubing, T. P. Van Doren, F. Sha, and M. J. Wilhelm, "Investigation of fundamental EMI source mechanisms driving common-mode radiation from printed circuit boards with attached cables," IEEE Trans. Electromagn. Compat., vol. 38, no. 4, pp. 557-566, Nov. 1996.

[8] C. Chen, "Examination of electronic module immunity using transfer functions," in IEEE Int. Symp. Electromagn. Compat. Dig., Aug. 2005, pp. $756-761$.

[9] K.-S. Chen, T.-S. Horng, C.-Y. Ho, J.-M. Wu, and K.-C. Peng, "Diagnosis of EMI to laptop WWAN device from TFT-LCD driver using non-contact measurement-based transfer function technique," in IEEE Int. Symp. Electromagn. Compat. Dig., Jul. 2010, pp. 301-304.

[10] Road Vehicles-Component Test Methods for Electrical Disturbances From Narrowband Radiated Electromagnetic Energy-Part 4: Bulk Current Injection (BCI), ISO Standard 11452-4, Apr. 2005.

[11] F. Grassi, F. Marliani, and S. A. Pignari, "Circuit modeling of injection probes for bulk current injection," IEEE Trans. Electromagn. Compat., vol. 49, no. 3, pp. 563-576, Aug. 2007.

[12] M. N. Iddagoda, N. Venkatarayalu, and Y. B. Gan, "Characterization, calibration and macromodeling of RF current probes," in Proc. AsiaPacific Microw. Conf., 2009, pp. 629-632.

[13] C. F. Chen, T. Y. Huang, and R. B. Wu, "Novel compact net-type resonators and their application to microstrip bandpass filters," IEEE Trans. Microw. Theory Tech., vol. 54, no. 2, pp. 755-762, Feb. 2006.

[14] J. S. Hong and M. J. Lancaster, Microstrip Filter for RF/Microwave Application. New York: Wiley, 2001.

[15] Y. Kayano, M. Tanaka, J. L. Drewniak, and H. Inoue, "Common-mode current due to a trace near a PCB edge and its suppression by a guard band," IEEE Trans. Electromagn. Compat., vol. 46, no. 1, pp. 46-53, Feb. 2004.

[16] F-150 Injection Current Probe Characterization. Torrance, CA: Fischer Custom Communications, Inc., 2006.

[17] C. R. Paul, "A comparison of the contributions of common-mode and differential-mode currents in radiated emissions," IEEE Trans. Electromagn. Compat., vol. 31, no. 2, pp. 189-193, May 1989. 